\title{
Asociación entre distintas medidas de Composición Corporal y Factores de Riesgo Cardiovascular en población adulta
}

\author{
Pablo Franquelo Moralesa, Sandra Serrano Martínez ${ }^{b}$, Pablo Moya Martínez $^{c}$, Jesús Buendía Bermejo ${ }^{\text {, }}$ \\ Mairena Sánchez López ${ }^{c}$, Montserrat Solera Martínez ${ }^{c}$, Blanca Notario Pacheco ${ }^{\circ}$
}

\begin{abstract}
a Servicio de Urgencias. Hospital Virgen de la Luz. Cuenca.

\section{b Servicio de Análisis Clínicos. Hospital Virgen de la Luz. Cuenca. \\ 'Centro de Estudios Socio- Sanitarios. CESS. Cuenca. \\ ${ }^{\mathrm{d}}$ Centro de Salud. Cuenca II. Cuenca.}

\section{Correspondencia:}

Pablo Franquelo Morales.

Servicio de Urgencias,

Hospital Virgen de la Luz, $\mathrm{C} /$ Hermandad de Donantes de Sangre $n^{\circ} 1$, 16002-Cuenca. Telf.: 969179900 ,

e-mail: pranquelo@yahoo.es

Recibido el 4 de marzo de 2008.

Aceptado para su publicación el 12 de abril de 2008.

\section{RESUMEN}

Objetivo. Evaluar el grado de acuerdo entre los indicadores de exceso de grasa corporal obtenidos mediante el cálculo de parámetros antropométricos, analíticos y con bioimpedanciómetro eléctrico de 4 y 8 electrodos.

Diseño. Estudio observacional transversal.

Emplazamiento. Centro de salud urbano de la ciudad de Cuenca.

Participantes. Se seleccionó una muestra aleatoria de 110 individuos entre 25 y 60 años mediante petición de colaboración voluntaria, durante los meses de marzo y abril de 2007.

Mediciones principales. Se recogieron variables sociodemográficas (edad y sexo), antropométricas [peso, talla, perímetro de cintura (PC), perímetro de cadera $(\mathrm{PCa})$ y pliegue tricipital $(\mathrm{PCT})$ ], analíticas [colesterol total, HDL-colesterol, LDL-colesterol, triglicéridos (TG), Apo A1, Apo B, glucemia basal e insulina basal] y porcentaje de grasa corporal obtenida mediante bioimpedanciómetro eléctrico de 4 (BIO 4) y 8 electrodos (BIO 8).

Resultados. No se observaron diferencias estadísticamente significativas en los parámetros analíticos del perfil lipídico (Colesterol total, HDL y LDL) entre los 2 grupos. En los individuos con resistencia a la insulina (RI) se observaron valores significativamente superiores de glucemia basal $(110,9 \pm 22,5$ frente a $98,9 \pm 19,08 \mathrm{mg} / \mathrm{dL})$ y de insulina basal $(24,3 \pm 12,5$ frente a $8,5 \pm 2,92 \mathrm{mg} / \mathrm{dL})$. La Rl correlacionó positiva y significativamente con el porcentaje de grasa corporal obtenido por $\mathrm{BIO} 4(r=0,31)$, por BIO $8(r=0,26)$ y con el índice de cintura altura (ICA; $r=0,22)$.

Conclusiones. En la práctica clínica la determinación del exceso de grasa corporal y RI por BIO 4 parece mejor que con $\mathrm{BIO} 8$, mostrando una alta fiabilidad entre ambos. No obstante, el ICA se presenta como un buen método de estimación de RI, además de resultar fácil, cómodo y accesible en las consultas de Atención Primaria.

Palabras clave. Obesidad, Resistencia a la Insulina.

\section{ABSTRACT}

Association between different measures of body composition and cardiovascular risk factors in the adult population

Objective. To assess the degree of agreement between fat indicators obtained by calculating anthropometric and analytical parameters and measurements with an electric impedance meter with 4 and 8 electrodes.

Design. Observational, cross-sectional study.

Setting. Urban health centre of the city of Cuenca.

Subjects. A random sample of 110 individuals between 25 and 60 years was selected through voluntary petition for collaboration, during the months of March and April 2007.

Main measurements. Socio-demographic (age and gender), anthropometric: (weight, height, waist circumference, hip circumference and tricipital fold (PCT)) and analytical variables (total cholesterol, HDL-cholesterol, LDL-cholesterol, triglycerides(TG), Apo A1, Apo B, basal glucose levels and basal insulin) and \% body fat obtained by an impedance electric meter with 4 and 8 electrodes were recorded.

Results. There were no statistically significant differences in the analytical parameters of the lipid profile (total cholesterol, HDL and LDL) between the 2 groups. There were significantly higher basal glucose levels $(110.9 \pm 22.5$ versus $98.9 \pm 19.08 \mathrm{mg} / \mathrm{dL})$ and basal insulin levels $(24.3 \pm 12.5$ versus $8.95 \pm 2.92 \mathrm{mg} / \mathrm{dL}$ ) in individuals with insulin resistance (IR). IR correlated positively and significantly with \% body fat obtained by 4 electrodes $(r=0.31), 8$ electrodes $(r=0.26)$ and waist $/$ height ratio $(r=0.22)$.

Conclusions. In clinical practice, the determination of excess body fat and IR with BIO 4 seems better than with $\mathrm{BIO} 8$, showing a high reliability between the two. However, waist height index (ICA) is presented as a good method for estimating IR, as well as being simple, convenient and accessible in Primary Care clinics.

Key words. Obesity, Insulin Resistance.

Presentado a los III Premios de Investigación en Atención Primaria, Cuenca 13 de diciembre del 2007. 


\section{INTRODUCCIÓN}

La obesidad constituye uno de los principales problemas de salud pública en los países desarrollados, y su prevalencia está aumentando en casi todo el mundo. En España el 17,5\% de las mujeres y el 13,2\% de los hombres de entre 25 y 60 años son obesos ${ }^{1-3}$.

El exceso de grasa corporal juega un papel central en el síndrome metabólico ${ }^{4}$, por su relación con los lípidos sanguíneos y con la presión arterial.

Tradicionalmente en la clínica el índice de masa corporal (IMC) se considera un buen indicador para definir la obesidad (y por tanto el exceso de grasa corporal), sin embargo, el IMC no es más que la relación entre el peso corporal y la talla. El peso corporal viene determinado tanto por la masa grasa como por la masa magra, por lo que en personas con gran desarrollo muscular la utilidad diagnóstica esta en entredicho.

Otros métodos antropométricos, como los pliegues cutáneos o el perímetro de cintura (PC), se han propuesto como medidas más adecuadas para estimar la grasa corporal.

Actualmente, la bioimpedancia eléctrica ha pasado a considerarse también como un método de estimación de la grasa corporal de utilidad clínica por su sencillez y reproducibilidad ${ }^{5,6}$.

El objetivo de este estudio es evaluar la relación entre los distintos indicadores de exceso de grasa corporal y los factores de riesgo cardiovascular.

\section{MATERIAL Y MÉTODO}

Estudio observacional transversal en una muestra de 110 sujetos de entre 25 y 60 años seleccionados aleatoriamente entre los demandantes de asistencia médica en el Centro de Salud Cuenca II de la ciudad de Cuenca durante los meses de marzo y abril de 2007.

A todas las personas que participaron en el estudio se les explicó detalladamente el objetivo del mismo y se les solicitó firmar un documento de consentimiento. El Comité Ético de Investigación Clínica del Hospital Virgen de la Luz de Cuenca aprobó el estudio.

\section{Variables antropométricas}

Se determinó el peso mediante báscula de lectura digital SECA $® 861$ redondeando a 100 gramos (gr), sin zapatos, llevando ropa ligera, de espaldas al registro de medida y en posición anatómica mediante dos mediciones. La talla mediante tallímetro incorporado a la balanza AÑÓ SAYOL $®$, en bipedestación, descalzo, con ropa ligera, perpendicular al suelo con los brazos descansados a los lados del cuerpo en posición erecta y cabeza ubicada en plano de Frankfort como la media de dos determinaciones ${ }^{1}$. El IMC fue calculado como el peso en kilogramos dividido entre el cuadrado de la talla en metros $\left(\mathrm{kg} / \mathrm{m}^{2}\right)$. Los participantes fueron clasificados como normopeso, sobrepeso y obesidad según criterios de la $\mathrm{OMS}^{7}$.

El pliegue tricipital (PCT) fue determinado como la media de tres determinaciones consecutivas realizadas mediante plicómetro HOLTAIN $®$ sobre el tríceps, en el punto medio entre el acromion y el olécranon, en la parte posterior del brazo izquierdo colocado en flexión de $90^{\circ}$.

El perímetro de cintura (PC) se midió en centímetros (cm) con una cinta métrica flexible, no extensible, de $0,5 \mathrm{~cm}$ de ancho $\times 2$ metros $(\mathrm{m})$ de largo con una exactitud de $0,1 \mathrm{~cm}$, en posición ortostática y espiración, alrededor de la cintura en el punto medio entre el último arco costal y la cresta iliaca y a un centímetro por debajo del ombligo, mediante la media de tres determinaciones. Se establecieron como valores de riesgo los establecidos por la SEEDO'1.

El Perimetro de Cadera ( $\mathrm{PCa}$ ) se midió en $\mathrm{cm}$, en el punto medio entre los trocánteres, a nivel del máximo relieve de los glúteos, coincidiendo con la sínfisis pubiana en la parte frontal del sujeto, calculando la media de tres determinaciones.

El índice cintura/cadera (ICC) se calculó mediante el cociente de las medias del perímetro de cintura y perímetro de cadera definiendo el valor de riesgo cardiovacular, en $\geq 1$ en hombres $y \geq 0,85$ en mujeres ${ }^{8}$. También se calculó el índice cintura/altura (ICA) y el índice cintura/triglicéridos (ICT).

La presión arterial se determinó mediante el monitor OMRON® M5-I, utilizando un brazal de 17-22 cm, 22$32 \mathrm{~cm}$ y $32-42 \mathrm{~cm}$, según el perímetro braquial ${ }^{9}$. Se practicaron tres lecturas en un periodo de 5 minutos y después de un mínimo de 5 minutos de descanso antes de la primera determinación. Los participantes estuvieron sentados, en un ambiente silencioso y tranquilo, con su brazo derecho apoyado a la altura del corazón ${ }^{10}$. Se les clasificó como hipertenso o no hipertenso según el protocolo de la JNC VII ${ }^{11}$.

\section{Variables analíticas}

Se obtuvieron de la extracción sanguínea de la vena cubital del antebrazo contrario al utilizado para la toma de la presión arterial tras ayuno de 12 horas y bajo condiciones estandarizadas. Se empleó el sistema al vacío VACUTAINER $R$ y tubo de $4,5 \mathrm{~mL}$, provisto de gel separador. Las muestras fueron procesadas en el laboratorio de Análisis Clínicos del Hospital Virgen de la Luz de Cuenca.

El colesterol total se obtuvo utilizando el método enzimático CHOD-PAP (colesterol-esterasa, colesterol-oxidasa, peroxidasa ${ }^{12}$. Los triglicéridos (TG) mediante el método enzimático GPO-PAP (glicerolfosfato-oxidasa, peroxidasa) ${ }^{13}$. El colesterol HDL (c-HDL) y colesterol LDL (c-LDL) se estimaron por método directo de $2^{a}$ generación, sin desproteinización, empleando enzimas modificadas por PEG y sulfato de dextrano ${ }^{14}$. Para la determinación de la apolipoproteina A-I (Apo A-I) y apolipoproteína $B(A p o B)$, se utilizó el test inmunoturbidimétrico Tina-quant $\AA$ versión 2 de Roche/Hitachi Modular Analitycs ${ }^{15}$. La dislipemia fue definida según normas SEEDO ${ }^{1}$ o en aquellos pacientes en tratamiento hipolipemiante ${ }^{16,17}$. El c-LDL fue calculado a partir de la fórmula de Friedewald ${ }^{18}$, o utilizando la medición directa cuando los TG superaban los 400 mg/dL.

La glucosa se analizó por método enzimático HK (hexocinasa) ${ }^{19}$. Se utilizó como control de calidad interno diario para cada parámetro los recomendados por la firma comercial Roche Diagnostics $\AA$, (Precinorm $U \circledast$,

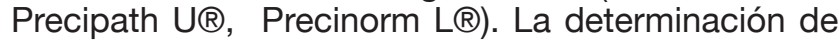
insulina se realizó por el método de inmunoquimiolu- 


\begin{tabular}{|c|c|c|c|c|}
\hline & $\mathbf{R I}$ & No RI & Total & p \\
\hline Número & 33 & 77 & 110 & \\
\hline Edad, años, media (DE) & $46,97(12,80)$ & $44,88(12,66)$ & $45,51(12,57)$ & NS \\
\hline \multicolumn{5}{|l|}{ Sexo, n (\%) } \\
\hline Varones & $18(36 \%)$ & $32(64 \%)$ & $50(46 \%)$ & NS \\
\hline Mujeres & $15(25 \%)$ & $45(75 \%)$ & $60(54 \%)$ & \\
\hline \multicolumn{5}{|l|}{ Presión arterial, mmHg, media (DE) } \\
\hline PAS & $139,27(17,70)$ & $128,54(17,58)$ & $131,89(18,10)$ & 0,040 \\
\hline PAD & $89,02(9,35)$ & $83,59(10,91)$ & $85,38(10,67)$ & 0,014 \\
\hline FC & $73,85(11,35)$ & $71,36(10,48)$ & $72,41(11,01)$ & NS \\
\hline \multicolumn{5}{|l|}{ Parámetros analíticos, media (DE) } \\
\hline Glucemia basal, mg/dL & $110,93(22,51)$ & $98,97(19,08)$ & $102,62(20,72)$ & 0,050 \\
\hline HOMA & $6,6(3,47)$ & $2,17(0,76)$ & $3,50(2,84)$ & $<0,001$ \\
\hline Insulina basal & $24,31(12,54)$ & $8,95(2,92)$ & $13,55(10,10)$ & 0,034 \\
\hline Colesterol total & $209,24(47,16)$ & $201,71(43,21)$ & $203,73(44,21)$ & NS \\
\hline $\mathrm{cHDL}$ & $50,84(18,27)$ & $60,59(14,57)$ & $57,49(16,35)$ & NS \\
\hline cLDL Directo & $126,36(38,15)$ & $114,64(32,66)$ & $118,47(34,80)$ & NS \\
\hline cLDL Calculado & $129,78(46,12)$ & $119,61(38,40)$ & $122,48(40,64)$ & NS \\
\hline $\mathrm{HbA1c}$ & $7,36(1,24)$ & $8,2(1,57)$ & $7,64(1,25)$ & NS \\
\hline Apo A1 & $142,84(33,09)$ & $154,27(24,68)$ & $150,84+-27,81$ & 0,048 \\
\hline Apo B & $99,9(24,46)$ & $88,38(26,28)$ & $91,84(26,18)$ & $<0,001$ \\
\hline Colesterol total/HDL & $4,52(1,57)$ & $3,52(1,25)$ & $3,83(1,42)$ & 0,010 \\
\hline Apo B/Apo A1 & $0,73(0,23)$ & $0,58(0,21)$ & $0,63(0,22)$ & 0,020 \\
\hline Triglicéridos & $170,06(133,28)$ & $109,05(63,03)$ & $127,53(93,25)$ & 0,001 \\
\hline \multicolumn{5}{|c|}{ Parámetros de la bioimpedancia eléctrica, media (DE) } \\
\hline$\%$ grasa corporal (4 electrodos) & $33,64(6,45)$ & $29,35(8,20)$ & $30,72(7,93)$ & 0,010 \\
\hline \% grasa corporal (8 electrodos) & $31,94(6,64)$ & $28,9(8,02)$ & $29,79(7,69)$ & NS \\
\hline \multicolumn{5}{|l|}{ Parámetros antropométricos, media (DE) } \\
\hline Talla & $164,72(10,11)$ & $165,48(9,49)$ & $165,33(9,58)$ & NS \\
\hline Peso & $79,09(16,26)$ & $71,8(14,74)$ & $74,27(15,53)$ & 0,023 \\
\hline IMC & $28,94(3,93)$ & $26,12(4,15)$ & $27,04(4,27)$ & 0,010 \\
\hline Pliegue tricipital & $17,34(6,91)$ & $16,76(6,67)$ & $16,98(6,66)$ & NS \\
\hline Perímetro cintura & $99,58(12,91)$ & $89,96(12,07)$ & $93,33(13,25)$ & 0,000 \\
\hline Índice cintura/cadera & $0,95(0,94)$ & $0,88(0,86)$ & $0,91(0,096)$ & 0,000 \\
\hline Índice cintura/altura & $0,6(0,076)$ & $0,54(0,72)$ & $0,56(0,079)$ & 0,000 \\
\hline Índice cintura/triglicéridos & $0,78(0,34)$ & $1(0,39)$ & $0,93(0,39)$ & NS \\
\hline Perímetro cadera & $104,21(7,65)$ & $101,22(7,49)$ & $102,26(7,67)$ & NS \\
\hline \multicolumn{5}{|l|}{ Diagnóstico, n (\%) } \\
\hline No hipertensos & $19(23,2 \%)$ & $63(76,8 \%)$ & $82(74,5 \%)$ & 0,007 \\
\hline Diabéticos & $9(75,0 \%)$ & $3(25 \%)$ & $12(10,9 \%)$ & $<0,001$ \\
\hline Fumadores & $5(21,7 \%)$ & $18(78,3 \%)$ & $23(20,9 \%)$ & NS \\
\hline No dislipemicos & $19(22,9 \%)$ & $64(77,1 \%)$ & $83(25,7 \%)$ & 0,004 \\
\hline Sobrepeso/Obesidad & $30(41,1 \%)$ & $43(58,9 \%)$ & $73(67,0 \%)$ & $<0,001$ \\
\hline Riesgo coronario, \% a 10 años, media (DE) & $1,45(2,15)$ & $0,84(1,37)$ & $1,02(1,65)$ & 0,140 \\
\hline
\end{tabular}

Tabla 1. Descripción de la muestra. RI: Sujetos con resistencia a la insulina; no RI: Sujetos sin resistencia a la insulina. PAS: Presión arterial sistólica; PAD: Presión arterial diastólica; FC: Frecuencia cardiaca; CT: colesterol total; cHDL: Colesterol HDL; CLDL directo: Coletesrol LDL directo; cLDL calculado: Colesterol LDL calculado; HbA1c: Hemoglobina glicosilada; Apo A1: Apolipoproteína A1; Apo B: Apolipoproteína B; TG: Triglicéridos; RC: Riesgo cardiovascular. 


\begin{tabular}{|c|c|c|c|c|c|c|c|c|c|c|c|c|c|c|c|c|}
\hline & PAS & PAD & $\mathrm{FC}$ & lb & Glucosa & HOMA & CT & CHDL. & $\begin{array}{c}\text { ColT otal/ } \\
\text { CHDL }\end{array}$ & $\begin{array}{r}\text { cLDL } \\
\text { Directo }\end{array}$ & $\begin{array}{c}\text { CLDL } \\
\text { Calculado }\end{array}$ & ApoA1 & ApoB & ApoB/A1 & TG & RC \\
\hline Peso & 0,264 & 0,357 & $-0,102$ & 0,060 & 0,088 & 0,084 & 0,103 & $-0,190$ & 0,264 & 0,126 & 0,015 & $-0,053$ & 0,197 & 0,243 & 0,429 & $-0,098$ \\
\hline$p$ & 0,010 & 0,000 & 0,333 & 0,568 & 0,403 & 0,422 & 0,324 & 0,069 & 0,011 & 0,228 & 0,884 & 0,612 & 0,058 & 0,019 & 0,000 & 0,348 \\
\hline IMC & 0,500 & 0,506 & $-0,077$ & 0,106 & 0,242 & 0,172 & 0,177 & $-0,228$ & 0,318 & 0,257 & 0,150 & $-0,058$ & 0,317 & 0,327 & 0,416 & 0,120 \\
\hline$p$ & 0,000 & 0,000 & 0,465 & 0,311 & 0,019 & 0,100 & 0,090 & 0,028 & 0,002 & 0,013 & 0,152 & 0,584 & 0,002 & 0,001 & 0,000 & 0,251 \\
\hline Cintura & 0,555 & 0,513 & $-0,081$ & 0,118 & 0,333 & 0,193 & 0,191 & $-0,269$ & 0,354 & 0,296 & 0,164 & $-0,100$ & 0,360 & 0,371 & 0,443 & 0,208 \\
\hline$p$ & 0,000 & 0,000 & 0,439 & 0,261 & 0,001 & 0,064 & 0,067 & 0,009 & 0,001 & 0,004 & 0,117 & 0,342 & 0,000 & 0,000 & 0,000 & 0,045 \\
\hline Indice cintura/cadera & 0,608 & 0,501 & $-0,085$ & 0,105 & 0,385 & 0,193 & 0,180 & $-0,273$ & 0,305 & 0,320 & 0,209 & $-0,107$ & 0,375 & 0,350 & 0,328 & 0,381 \\
\hline$p$ & 0,000 & 0,000 & 0,416 & 0,318 & 0,000 & 0,064 & 0,085 & 0,008 & 0,003 & 0,002 & 0,044 & 0,307 & 0,000 & 0,001 & 0,001 & 0,000 \\
\hline \multirow[t]{2}{*}{ Indice cintura/altura } & 0,634 & 0,537 & $-0,068$ & 0,132 & 0,381 & 0,221 & 0,219 & $-0,259$ & 0,346 & 0,347 & 0,233 & $-0,093$ & 0,396 & 0,382 & 0,380 & 0,322 \\
\hline & 0,000 & 0,000 & 0,516 & 0,207 & 0,000 & 0,033 & 0,035 & 0,012 & 0,001 & 0,001 & 0,024 & 0,375 & 0,000 & 0,000 & 0,000 & 0,002 \\
\hline Pliegue tricipital & 0,021 & 0,095 & 0,093 & 0,013 & $-0,027$ & 0,022 & 0,142 & $-0,012$ & 0,141 & 0,122 & 0,084 & 0,051 & 0,142 & 0,137 & 0,150 & $-0,107$ \\
\hline$p$ & 0,838 & 0,364 & 0,375 & 0,900 & 0,797 & 0,833 & 0,175 & 0,906 & 0,177 & 0,245 & 0,421 & 0,627 & 0,175 & 0,191 & 0,151 & 0,309 \\
\hline $\begin{array}{l}\text { \% Grasa corporal } \\
\text { (4 electrodos) }\end{array}$ & 0,456 & 0,455 & 0,080 & 0,331 & 0,207 & 0,313 & 0,187 & $-0,252$ & 0,364 & 0,268 & 0,167 & $-0,088$ & 0,323 & 0,351 & 0,398 & 0,211 \\
\hline$p$ & 0,000 & 0,000 & 0,458 & 0,002 & 0,051 & 0,003 & 0,080 & 0,017 & 0,000 & 0,011 & 0,118 & 0,411 & 0,002 & 0,001 & 0,000 & 0,047 \\
\hline $\begin{array}{l}\text { \% Grasa corporal } \\
\text { (8 electrodos) }\end{array}$ & 0,445 & 0,424 & 0,021 & 0,284 & 0,177 & 0,267 & 0,206 & $-0,242$ & 0,366 & 0,314 & 0,192 & $-0,071$ & 0,378 & 0,388 & 0,364 & 0,178 \\
\hline$P$ & 0,000 & 0,000 & 0,845 & 0,008 & 0,106 & 0,013 & 0,059 & 0,026 & 0,001 & 0,003 & 0,079 & 0,519 & 0,000 & 0,000 & 0,001 & 0,102 \\
\hline
\end{tabular}

Tabla 2. Análisis de correlación entre las diferentes formas de medir la obesidad y variables clínico-analíticas de interés (ajustadas por sexo). PAS: Presión arterial sistólica; PAD: Presión arterial diastólica; FC: Frecuencia cardiaca; lb: Insulina basal; CT: colesterol total; cHDL: Colesterol HDL; cLDL directo: Coletesrol LDL directo; cLDL calculado: Colesterol LDL calculado; Apo A1: Apolipoproteína A1; Apo B: Apolipoproteína B; TG: Triglicéridos; RC: Riesgo cardiovascular.

Resistencia a la insulina

Si (\%) No (\%) Chi-cuadrado $\quad$ gl $\quad$ p

\begin{tabular}{|c|c|c|c|c|c|}
\hline \multicolumn{6}{|l|}{ IMC* } \\
\hline Normopeso & $3(9,1 \%)$ & $33(46,4 \%)$ & \multirow{3}{*}{17,529} & \multirow{3}{*}{2} & \multirow{3}{*}{$<0,001$} \\
\hline Sobrepeso & $15(45,5 \%)$ & $32(42,1 \%)$ & & & \\
\hline Obesidad & $15(45,5 \%)$ & $11(14,5 \%)$ & & & \\
\hline \multicolumn{6}{|l|}{ Cintura } \\
\hline Normal & $6(15,8 \%)$ & $32(84,2 \%)$ & \multirow{3}{*}{12,051} & \multirow{3}{*}{2} & \multirow{3}{*}{0,002} \\
\hline Riesgo medio & $5(19,2 \%)$ & $21(80,8 \%)$ & & & \\
\hline Gran riesgo & $22(47,8)$ & $24(52,2 \%)$ & & & \\
\hline \multicolumn{6}{|c|}{ Indice cintura/cadera } \\
\hline Normal & $17(51,5 \%)$ & $53(68,8 \%)$ & \multirow{2}{*}{2,993} & \multirow{2}{*}{1} & \multirow{2}{*}{0,084} \\
\hline Riesgo & $16(48,5 \%)$ & $24(31,2 \%)$ & & & \\
\hline \multicolumn{6}{|c|}{$\%$ grasa corporal (4 electrodos) } \\
\hline Normopeso & $2(6,3 \%)$ & $16(23,2 \%)$ & \multirow{3}{*}{6,487} & \multirow{3}{*}{2} & \multirow{3}{*}{0,039} \\
\hline Sobrepeso & $3(9,4 \%)$ & $12(17,4 \%)$ & & & \\
\hline Obesidad & $27(84,4 \%)$ & $41(59,4 \%)$ & & & \\
\hline \multicolumn{6}{|c|}{$\%$ grasa corporal (8 electrodos) } \\
\hline Normopeso & $2(6,7 \%)$ & $15(23,8 \%)$ & \multirow{3}{*}{3,999} & \multirow{3}{*}{2} & \multirow{3}{*}{0,135} \\
\hline Sobrepeso & $4(13,3 \%)$ & $7(11,1 \%)$ & & & \\
\hline Obesidad & $24(80,0 \%)$ & $41(65,1 \%)$ & & & \\
\hline
\end{tabular}

Tabla 3. Asociación entre las diferentes mediciones y la resistencia a la insulina. * Puntos de corte de documento de consenso: obesidad y riesgo cardiovascular, J. Aranceta. IMC: Índice de masa corporal. 


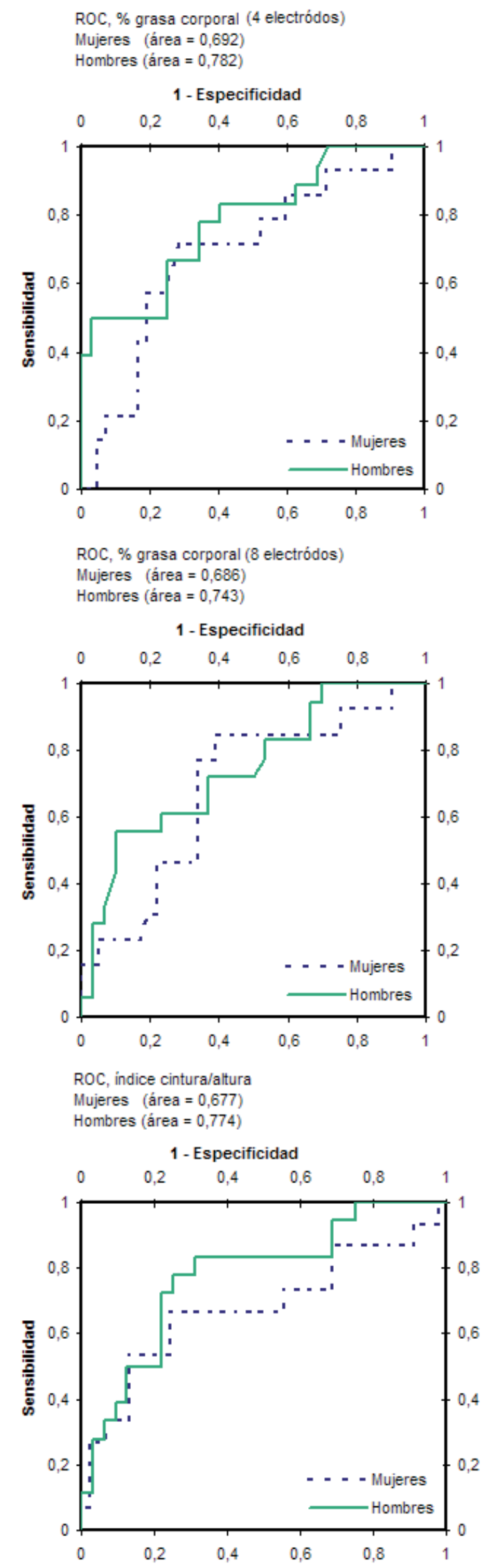

Figura 1. Curvas ROC. miniscencia, procesándose en el analizador IMMULITE 2000 de Siemens Medical Solutions Diagnostics $\mathbb{R}^{20}$ y utilizando los calibradores y controles de calidad recomendados por la firma comercial. A partir de los valores obtenidos se calculó el índice de la resistencia a la insulina (RI) según modelo Homeostasis Model Assessment (HOMA) ${ }^{22}$. Definimos RI si HOMA era $\geq$ $3,8^{23}$. Para la clasificación de la diabetes, se siguieron las recomendaciones de la $\mathrm{OMS}^{7,21}$.

\section{Porcentajes de grasa corporal}

Se estimaron mediante el impedanciómetro BC-420 MA y el BC-418 (TANITA Corp., Tokio, Japan) ${ }^{24,25}$, de 4 y 8 electrodos respectivamente, redondeando a 1 $\mathrm{Kg}$ correspondiente al peso de la ropa incluida en la medición. Se calculó una media de dos 2 lecturas en condiciones de temperatura y humedad controladas, en reposo de al menos quince minutos y tras micción previa, con el paciente descalzo, en ropa ligera, con brazos separados de tórax. Este dispositivo utiliza la dual energy X-ray absortiometry (DXA) como método de referencia y calcula la composición corporal basándose en estimaciones derivadas del análisis de regresión con las variables talla, peso y sexo.

\section{Análisis estadístico}

Para las variables cualitativas se determinaron los porcentajes y sus intervalos de confianza (IC) del 95\%. En las variables cuantitativas se estudió la media \pm DE y los IC del 95\%. La normalidad se estudió mediante la prueba de Kolmogorov-Smirnov. Se utilizó la prueba T-Student para la comparación de medias, el estadístico $\mathrm{X}^{2}$ de Pearson para estudiar la asociación entre las diferentes medidas y la RI. El coeficiente de correlación de Pearson o Spearman, según la naturaleza de las variables y ajustando por sexo, se empleó para medir el grado de asociación entre los diferentes métodos de estimación del exceso de grasa corporal y los factores de riesgo cardiovascular. Se diseñaron curvas "Receiver Operating Characteristic Curves" (Curvas ROC), para predecir los puntos de corte tanto del \% de grasa corporal determinado por $\mathrm{BIO} 4$ y $\mathrm{BIO}$ 8, como de ICA, a partir de los cuales aumenta el riesgo de padecer RI. La significación estadística fue establecida con $p \leq 0,05$. Todos los análisis se realizaron con el paquete estadístico SPSS para Windows $®$, versión 15.0. (SPSS Inc.).

\section{RESULTADOS}

Participaron en el estudio 110 personas, con una edad media 45,5 $\pm 12,5$ años, de los cuales el $54 \%$ eran mujeres. Las características antropométricas, clínicas y bioquímicas se resumen en la tabla 1 .

No existieron diferencias significativas en la edad y los parámetros analíticos del perfil lipídico (HDL, Colesterol total y LDL) entre los individuos con RI y sin RI. En cambio, sí que se observaron en la glucemia basal (RI: 110,9 \pm 22,5; no RI: 98,9 $\pm 19,1)$, insulina basal (Rl: $24,3 \pm 12,5$; no RI: $8,9 \pm 2,9)$, PAS (RI: $139,3 \pm 17,7$; no RI: $128,5 \pm 17,6), \operatorname{PAD}(\mathrm{Rl}: 89,0 \pm 9,4$; no RI: 83,6 \pm

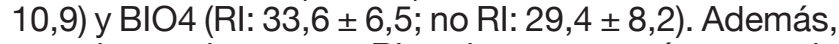
entre los pacientes con RI se detectaron más casos de dislipemia, con mayor concentración plasmática de TG 
y Apo A, y con diabetes (75\%). Entre los pacientes sin RI hubo más no hipertensos $(76,8 \%)$, no dislipémicos $(77,1 \%)$ y personas con sobrepeso/obesidad $(58,9 \%)$. No se observaron diferencias significativas entre los dos grupos en el cálculo del riesgo coronario a los 10 años según tabla SCORE ${ }^{25}$.

En la tabla 2 se muestra la correlación entre las diferentes formas de medir la obesidad y las variables clínico-analíticas de interés ajustadas por sexo. La RI se correlaciona positiva y significativamente con el \% de grasa estimado con $\mathrm{BIO} 4(r=0,31)$, con $\mathrm{BIO} 8(r$ $=0,26)$ e ICA $(r=0,22)$. La insulinemia basal sigue un patrón similar a la RI, cuando el $\%$ de grasa es obtenido con $\mathrm{BIO} 4(r=0,33 ; \mathrm{p}=0,002)$ y con $\mathrm{BIO} 8(r=0,28 ; p$ $=0,008$ ). No encontramos correlación en las variables ICC, PCT e IMC, sin embargo existe una correlación positiva y significativa entre el PC y los TG.

La tabla 3 representa la asociación entre la RI y las diferentes variables categorizadas. Se obtiene significación estadística entre las variables \% de grasa corporal estimado con $\mathrm{BIO} 4$, IMC y PC, mostrando una mayor fuerza de asociación entre las dos últimas.

En la figura 1 se muestran las curvas ROC por sexo (1) para el punto de corte de \% de grasa corporal determinado por $\mathrm{BIO} 4$, (2) por $\mathrm{BIO} 8$ y (3) para el ICA a partir de las cuales se produce RI. En las tres variables estudiadas, las mayores áreas bajo la curva se observaron en los hombres. El punto de corte para la variable \% de grasa corporal determinada con $\mathrm{BIO}$ 4 en los hombres fue 27,25 ( $S=77,8 \%, E=34,3 \%$ ), con un área bajo la curva de 0,78 , y $36,67(S=64,3 \%$, $\mathrm{E}=74 \%$ ) en las mujeres. Respecto a la variable \% de grasa corporal determinada con $\mathrm{BIO} 8$, se estableció el punto de corte en 26,77 ( $S=72,2 \%, E=36,6 \%$ ) en los hombres, con un área bajo la curva de 0,74, y en 36 ( $S=76,9 \%, E=34 \%$ ) en las mujeres. La variable ICA obtuvo un área bajo la curva de 0,77 , con un punto de corte de $0,59(\mathrm{~S}=77,8 \%, \mathrm{E}=24,9 \%)$ en los hombres y $0,58(S=66,7 \%, E=24,3 \%)$ en las mujeres.

\section{DISCUSIÓN}

Existe una relación inequívoca entre el exceso de grasa corporal y los factores de riesgo cardiovascular y metabólico en la población adulta. La obesidad provoca $\mathrm{RI}$, definida por un índice $\mathrm{HOMA} \geq 3.8$, lo que constituye el núcleo principal del síndrome metabólico. En nuestro estudio se definió este parámetro y se obtuvo una relación con las distintas formas de medir el exceso de grasa.

Debido a que el IMC y el porcentaje de grasa corporal varían según la población estudiada, la utilización de la bioimpedancia eléctrica como nuevo determinante de grasa corporal esta ampliamente difundida ${ }^{26}$. Su fácil utilización, fiabilidad y no invasividad hacen de ella una herramienta interesante para estudios epidemiológicos.

La determinación de \% grasa por $\mathrm{BIO} 4$, BIO 8 e ICA, presentaron correlación positiva, y estadísticamente significativa con los valores del índice HOMA. Se aprecia una correlación estadísticamente significativa entre el PC y los TG plasmáticos $(r=0,443)$, apoyando el concepto de "cintura hipertrigliceridémica ${ }^{27}$ ya descrito en otros estudios.
En nuestro estudio, el ICA fue el mejor parámetro antropométrico para la estimación de la $\mathrm{Rl}(r=0,22 ; p=$ $0,003)$. Además, se correlaciona con los parámetros analíticos de perfil lipídico. Unido todo ello a su simplicidad y fácil obtención, hacen del ICA una medida útil en las consultas de Atención Primaria (AP). Los pacientes con RI no presentaron un elevado ICC, como se demuestra en estudios recientes ${ }^{28}$.

Por otro lado, se ha constatado que el PT no resulta práctico en la clínica diaria debido a la dificultad para la medición de grandes pliegues, ya que mide únicamente la grasa subcutánea y a la variabilidad interobservador, lo que lo convierte en un método más interesante para la población infantil ${ }^{29}$.

No se encontró significación en cuanto al riesgo coronario entre individuos con y sin RI. Cuando intentamos predecir la RI a partir de los parámetros de ICA y \% de grasa determinado por $\mathrm{BIO} 4$ y $\mathrm{BIO} 8$, se constata una mayor especificidad en este último método $(36,6 \%)$ para hombres, sin apenas diferencias en mujeres (34\%). No obstante, la muestra analizada es pequeña, por lo que no resulta posible inferir causalidad, sino evaluar resultados en función de la asociación de diferentes variables.

En conclusión, la determinación del exceso de grasa corporal y $\mathrm{RI}$ por $\mathrm{BIO} 4$ parece mejor que con BlO 8, mostrando una alta fiabilidad entre ambos. No obstante, el ICA se presenta como un buen método de estimación de RI, además de resultar fácil, cómodo y accesible en las consultas de AP.

\section{BIBLIOGRAFÍA}

1. Salas-Salvadó J, Rubio MA, Barbany M, Moreno B; Grupo Colaborativo de la SEEDO.SEEDO 2007 Consensus for the evaluation of overweight and obesity and the establishment of therapeutic intervention criteria. Med Clin (Barc). 2007; 128:184-96.

2. Gutiérrez-Fisac JL, Regidor E, Banegas JR, Rodríguez Artalejo F.Prevalence of obesity in the Spanish adult population: 14 years of continuous increase. Med Clin (Barc). 2005; 124:196-197.

3. Aranceta-Bartrina J, Serra-Majem L, Foz-Sala M, MorenoEsteban B. Grupo Colaborativo SEEDO. Prevalence of obesity in Spain. Med Clin (Barc). 2005; 125:460-466.

4. Reaven GM. Banting lectura 1988: role of insulin resistance in human disease. Diabetes. 1988; 37:1595-607.

5. Jia WP, Lu JX, Xiang KS, Bao YQ, Lu HJ, Chen L. Prediction of abdominal visceral obesity from body mass index, waist circumference and waist-hip ratio in Chinese adults: receiver operating characteristic curves analysis. Biomed Environ Sci. 2003; 16:206-211.

6. Lukaski HC. Regional bioelectrical impedance analysis: applications in health and medicine. Acta Diabetol. 2003; 40:196-199.

7. WHO. Programme of Nutrition, Family and Reproductive Health. Obesity. Preventing and managing the global epidemic. Report of a WHO consultation on obesity. Geneva, 3-5 June, 1997. Geneva: WHO; 1998.

8. Aranceta J, Foz M, Gil B, Jover E, Mantilla T, Millan J, Monereo S, Moreno B. Documento de consenso: obesidad y riesgo cardiovascular. Clin Invest Arterioscl. 2003; 15:196233.

9. El Assaad MA, Topouchian JA, Asmar RG. Evaluation of two devices for self-measurement of blood pressure according to the international protocol: the Omron M5-I and the Omron 705IT. Blood Press Monit. 2003; 8:127-133.

10. O’Brien E, Waeber B, Parati G, Staessen J, Myers MG. Blood pressure measuring devices: recommendations of the European Society of Hypertension. BMJ 2001; 322: 531-536. 
11. The Seventh Report of the Joint Nacional Committee on Prevention, Detection, Evaluation, and Treatment of High Blood Pressure (JNC 7): is it really practical? Natl Med J India. 2004; 17:227.

12. Zak B. Colesterol methodologies: a review. Clin Chem. 1977; 23:1201-1214.

13. Shephard MDS, Whiting MJ. Falsely low estimation of triglycerides in lipemic plasma by the enzymatic triglyceride method with modified trinder's chromogen. Clin Chem. 1990; 36:325-329.

14. Sugiuchi H, Uji Y, Okabe H, Irie T, Uekama K, Kayahara N, Miyauchi K. Direct measurement of high-density lipoprotein cholesterol in serum with polyethylene glycol-modified enzymes and sulfated alpha-cyclodextrin. Clin Chem. 1995; 41:717-723.

15. Rifai N, King ME. Inmunoturbidimetric Assays of Apolipoproteins A, Al, All and B in serum. Clin Chem. 1986; 32:957961.

16. Carr M, Brunzell JD. Abdominal obesity and dyslipidemia in the metabolic syndrome: importance of type 2 diabetes and familial combined hyperlipidemia in coronary artery disease risk. J Clin Endocrinol Metab. 2004; 89:2601-2607.

17. Luengo E. Las dislipemias como factor de riesgo. Definición, clasificación y diagnóstico. En: Del Río A., De Pablo C., editores. Manual de cardiología preventiva. Ed. SCM Sociedad Española de Cardiología. 2005. p. 57-71.

18. Friedewald WT, Levi RI, Fredrickson DS. Estimation of the concentration of low density lipoproteins cholesterol in plasma without use of the ultracentrifuge. Clin Chem. 1972; 18:499-502.

19. Greiling H, Gressner AM, eds. Lehrbuch der Klinischen Chemie und Pathobiochemie, 3rd ed. Stuttgard/New York: Schauttauer Verlag; 1995.

20. Schade DS, Eaton RP, Spencer W. Normalization of plasma insulin profiles in diabetic subjects with programmed insulin delivery. Diabetes care 1980; 3:9-14

21. Matthews DR, Hosker JP, Rudenski AS, Naylor BA, Treacher DF, Turner RC. Homeostasis model assessment: insulin resistance and beta-cell function from fasting plasma glucose and insulin concentrations in man. Diabetologia. 1985; 28:412-419.

22. Ascaso JF, Romero P. Cuantificación de insulinorresistencia con los valores de insulina basal e índice HOMA en una población no diabética. Med Clin (Barc) 2001; 117:530-553.

23. The Expert Committee on the Diagnosis and Classification of Diabetes Mellitus. Report of the Expert Committee on The Diagnosis and Classification of Diabetes Mellitus. Diabetes Care 2000; 23:S4-S19.

24. Sun SS, Chumlea WC, Heymsfield SB, Lukaski HC, Schoeller D, Friedl K, Kuczmarski RJ, Flegal KM, Johnson CL, Hubbard VS. Development of bioelectrical impedance analysis prediction equations for body composition with the use of a multicomponent model for use in epidemiologic surveys. Am J Clin Nutr 2003; 77:331-340.

25. Sans S, Fitzgerald AP, Royo D, Conroy R, Graham I. Calibrating the SCORE cardiovascular risk chart for use in Spain. Rev Esp Cardiol. 2007; 60:476-485.

26. Pietrobelli A, Rubiano F, St-Onge MP, Heymsfield SB. New bioimpedance analysis system: improved phenotyping with whole-body analysis. Eur J Clin Nutr. 2004; 58:14791484.

27. Lemieux I, Pascot $A$, Couillard $C$, Lamarche $B$, Tchernof $A$ Alméras N, Bergeron J, Gaudet D, Tremblay G, Prud'homme D, Nadeau A, Després JP. Hypertriglyceridemic waist: A marker of the atherogenic metabolic triad (hyperinsulinemia; hyperapolipoprotein B; small, dense LDL) in men? Circulation. 2000; 102:179-184.

28. Molist-Brunet N, Jimeno-Mollet J, Franch-Nadal J. Correlación entre las diferentes medidas de obesidad y el grado de resistencia a la insulina. Aten Primaria. 2006; 37:30-36.

29. Martínez Vizcaíno V, Salcedo Aguilar F, Franquelo Gutiérrez R, Torrijos Regidor R, Morant Sánchez A, Solera Martínez $\mathrm{M}$, Rodríguez Artalejo F. Prevalence of obesity and trends in cardiovascular risk factors among Spanish school children, 1992-2004: the Cuenca (Spain) study. Med Clin (Barc). 2006; 126:681-685. 